\title{
merging art and neuroscience
}

I am an artist that creates art that celebrates the beauty, complexity and mystery of the brains of those individuals who have one of the most common neurological diseases of the human body, multiple sclerosis (MS). My art is based on the latest advances in neuro-imaging technology, which are largely unavailable to people outside the scientific community, gaining access to these technologies by working with the assistance of leaders in the fields of neurology and neuroscience.

I create etchings and digital prints based on MRI scans of my own brain, transforming them into intimate portraits of beauty, complexity and mystery. My work features rich color that give the brain's structures the quality of dream images, revealing the humanity behind the clinical images.

Access to these state-of-the-art technologies allows me to creatively display parts of the brain in ways rarely seen before My aim as an artist is to create art that explores the splendor of the brain with all of its powers to change, adapt and confound.

This quest to understand the brain was inspired by my diagnosis in 1991 of Multiple Sclerosis, a diagnosis that ended my career as a lawyer dedicated to working with the poor. But, to my amazement, it allowed me to discover myself as an artist who is able to offer my art to the general public as well as to members of these scientific, medical and the disabled community.

Neurologists diagnose and track the disease of MS through magnetic resonance images of the brain (MRIs). My diagnosis of MS had initiated a deep fascination with the brain and I soon began to creatively explore this remarkable organ. I became focused on the magnetic resonance images(MRIs) of my brain, which were frightening yet mesmerizing. I felt a strong urge to reinterpret these images-to use them to explore the wonder and beauty of all brains including those with a disease. I further realized that I wanted my images to create an artist's view of imaging technology-one that is accessible to those who view these revealing pictures as either subject or doctor or scientist and also one that captures some of the feelings and emotions evoked by these kind of medical images.

My life with MS includes being constantly confronted with scans of my changing brain. Because of my unique intimacy with brain scans, I am familiar with the vulnerability that is associated with having a scans of my naked brain - with being exposed from the inside out. Through my art, I share the insights I have gained by witnessing the newest brain imaging with the scientific and general public. My art work intends to form of a new kind of visual imagery, one that interprets a structure where creativity, personality, and selfhood reside alongside disease reminding viewers that this grey tissue and the body that shelters it represent far more than illness and human imperfection.

My work is in the permanent collection of the Harvard University Center for Brain Science; Johns Hopkins University; University of California, Berkeley; Center for Mind and Brain, University of California, Davis; the Basque Neuroscience Institute, San Sebastian, Spain. It is featured in a documentary filmed by National Public Television aired throughout the United States.

On a personal note, when I was diagnosed with MS, I was playing with my two young children at the park when I suddenly lost the ability to speak. When I went to the hospital, they immediately performed a brain biopsy which indicated that my brain had evidence of demyelinating disease. Since that episode, which is extremely unusual for MS, I have experienced much more typical MS symptoms. Several months ago, my neurologist characterized my disease as progressive MS I use a wheelchair to ambulate and have limited use of my hands. Despite these setbacks, I am passionately involved in creating art that makes medical technology more accessible to the public. I feel lucky and grateful that I still have the ability to produce images that I can offer the public.

It takes a village to thrive when you are living a chronic progressive disease. My resilience stems from the fact that I have a husband who has stood by me throughout the years of my illness and that I have two wonderful boys who keep me laughing.

$$
\text { doi : 10.5214/ans.0972.7531.12190202 }
$$

\section{Elizabeth Jameson}

Public Interest Artist

Transforming Medical Imaging into

Fine Art

San Francisco Bay Area

USA 